\title{
Orientation dependence of the magnetoelastic coupling constants in strained FCC Co and Ni: an ab initio study
}

\author{
G.Y. Guo* \\ Department of Physics, National Taiwan University, 1 Sec. 4, Roosevelt Road, Taipei, Taiwan 106, ROC
}

\begin{abstract}
First-principles magnetocrystalline anisotropy energy calculations for FCC Co and Ni strained uniaxially along the

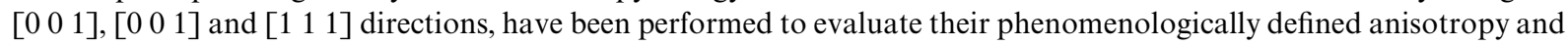
magnetoelastic constants. In the cubic limit, the calculated magnetoelastic coupling constant $B_{1}$ of Co and $B_{2}$ of Ni agree well with experiments. The calculated $\operatorname{Co} B_{1}$ and $B_{2}$ show a pronounced dependence on both the strain size and orientation whereas that of Ni remain almost constant in the strain region below $2.5 \%$. C) 2000 Elsevier Science B.V. All rights reserved.
\end{abstract}

Keywords: Magnetic anisotropy; Magnetoelastic coupling

The magnetic anisotropy energy of a magnet is defined as the energy difference between the easy and a hard magnetization axes. The magnetic anisotropy energy consists of the magnetocrystalline anisotropy energy (MAE) of the electronic origin and the magnetostatic anisotropy energy due to magnetic dipole interaction in the material. In a layered magnetic structure, the magnetic dipole energy always prefers an in-plane magnetization and a possible perpendicular anisotropy must come from the magnetocrystalline anisotropy in the electronic structure via the spin-orbit coupling. Extensive experimental and theoretical studies have been carried out on the magnetic multilayers to understand the key factors which determine the magnetocrystalline anisotropy of these systems (see, e.g. Ref. [1]). This is partly due to the fact that a magnetic multilayer with a perpendicular magnetization is useful in technological applications such as high-density magneto-optical recording and memory devices. It has become clear that the reduced dimensionality and symmetry at the surface and interface in a multilayer can result in a perpendicular anisotropy which is 2-3 orders of magnitude larger than in the corresponding

* Tel.: + 886-2-23626937; fax: + 886-2-23639984.

E-mail address: gyguo@phys.ntu.edu.tw (G.Y. Guo) bulk solids [1]. It has been found that the perpendicular anisotropy can also be caused by the lattice mismatch strain via magnetoelastic coupling in a multilayer system (see, e.g. Refs. [2,3]). Thus, the consequences of lattice mismatch strains and the magnetoelastic anisotropy contributions that they induce have been the subject of a number of recent experimental and theoretical studies (see, e.g. Refs. [2-7]).

For the cubic systems, the magnetoelastic energy is

$$
\begin{aligned}
E= & B_{1}\left(\varepsilon_{11} \beta_{x}^{2}+\varepsilon_{22} \beta_{y}^{2}+\varepsilon_{33} \beta_{z}^{2}\right) \\
& +2 B_{2}\left(\varepsilon_{12} \beta_{x} \beta_{y}+\varepsilon_{23} \beta_{y} \beta_{z}+\varepsilon_{31} \beta_{z} \beta_{x}\right) .
\end{aligned}
$$

Here $\beta_{x}, \beta_{y}, \beta_{z}$ are the direction cosines of the magnetization relative to the crystalline axes, and $\varepsilon_{i j}$ are the strain matrix elements. $B_{1}$ and $B_{2}$ are the magnetoelastic coupling constants of the solid. We have recently calculated the magnetocrystalline anisotropy energy of FCC Co as

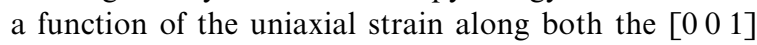
and $\left[\begin{array}{lll}1 & 1 & 0\end{array}\right]$ directions by using a first-principles relativistic electronic band structure method [8]. From the calculated anisotropy energies, we were able to evaluate the magnetoelastic coupling constant $B_{1}$ which shows a pronounced dependence on the uniaxial strain size and orientation. The purpose of the present paper is, first, to extend this work to the [ $\left[\begin{array}{lll}1 & 1 & 1\end{array}\right]$ uniaxial strain to study the strain orientation dependence of the magnetoelastic 
coupling constant $B_{2}$ and second, to report similar calculations performed for $\mathrm{Ni}$ whose magnetic multilayers show a range of interesting behaviors especially double spin-orientation transition [2,3,7].

In this paper, we consider a strained, infinite crystal. We study the unit cell of FCC Co and Ni for the three lattice types that correspond to the FCC $\left[\begin{array}{lll}1 & 1 & 0\end{array}\right],\left[\begin{array}{lll}0 & 0 & 1\end{array}\right]$ and [ $\left[\begin{array}{lll}1 & 1 & 1\end{array}\right]$ orientations. These are the orthorhombic, tetragonal and trigonal lattices, respectively. The strain is then incorporated by varying the $c / a$ lattice parameter ratio in each case.

The $\left[\begin{array}{lll}1 & 1 & 0\end{array}\right]$ strain induces both a uniaxial anisotropy $\left(K_{\text {out }}\right)$ along [ $\left[\begin{array}{lll}1 & 1 & 0\end{array}\right]$ and a further anisotropy in the $\left[\begin{array}{lll}0 & 0 & 1\end{array}\right]-\left[\begin{array}{lll}1 & 1 & 0\end{array}\right]$ plane $\left(K_{\text {in }}\right)$ [8]. These anisotropy constants $\left(K_{\text {out }}, K_{\text {in }}\right)$ can be expressed in terms of the difference in total energy for the magnetization along four different crystallographic directions [8],

$$
\begin{aligned}
& K_{\text {out }}=E_{\left[\begin{array}{lll}
1 & \overline{1} & 0
\end{array}\right]}-E_{\left[\begin{array}{lll}
1 & 1 & 0
\end{array}\right]},
\end{aligned}
$$

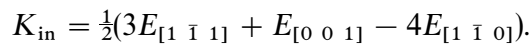

Throughout this paper we shall neglect the tiny cubic anisotropy constant $K_{1} . K_{\text {out }}$ and $K_{\text {in }}$ depend on the spin-orbit coupling to second order whereas $K_{1}$ is a fourth-order term. Both the $\left[\begin{array}{lll}0 & 0 & 1\end{array}\right]$ and $\left[\begin{array}{lll}1 & 1 & 1\end{array}\right]$ strains induce a uniaxial anisotropy $K_{\text {out }}$ along the $\left[\begin{array}{lll}0 & 0 & 1\end{array}\right]$ and [ $\left.\begin{array}{lll}1 & 1 & 1\end{array}\right]$ directions, respectively. The equivalent expression to Eqs. $(2 \mathrm{a})$ and $(2 \mathrm{~b})$ are now for the $\left[\begin{array}{lll}0 & 0 & 1\end{array}\right]$ orientation:

$K_{\text {out }}=E_{\left[\begin{array}{lll}1 & 0 & 0\end{array}\right]}-E_{\left[\begin{array}{lll}0 & 0 & 1\end{array}\right]}$

and for the [ $\left[\begin{array}{lll}1 & 1 & 1\end{array}\right]$ orientation:

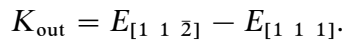

From Eqs. (1a) and (1b), we can express the magnetoelastic coupling constants in terms of the calculated anisotropy constants and the lateral strains (the elastic strains in the plane perpendicular to the uniaxial orientation) as follows. For the $\left[\begin{array}{lll}0 & 0 & 1\end{array}\right]$ orientation,

$B_{1}=\frac{1}{3} K_{\text {out }} / \varepsilon_{11}$,

where elastic strain element $\varepsilon_{11}=\varepsilon$ (the lateral elastic strain). For the $\left[\begin{array}{lll}1 & 1 & 1\end{array}\right]$ orientation,

$B_{2}=-\frac{1}{3} K_{\text {out }} / \varepsilon_{12}$,

where elastic strain element $\varepsilon_{12}=-2 \varepsilon$. For the $\left[\begin{array}{lll}1 & 1 & 0\end{array}\right]$ orientation,

$$
\begin{aligned}
& B_{1}=-\frac{1}{3}\left(K_{\text {in }}+\frac{1}{2} K_{\text {out }}\right) / \varepsilon_{11}, \\
& B_{2}=-\frac{1}{2} K_{\text {out }} / \varepsilon_{12},
\end{aligned}
$$

where $\varepsilon_{11}=-\left(\frac{1}{2}\right) \varepsilon$ and $\varepsilon_{12}=-3 \varepsilon$. Thus, from the calculated $K_{\text {out }}$ and $K_{\text {in }}$, we can obtain the FCC Co and Ni $B_{1}$ and $B_{2}$ as a function of the lateral strain $\varepsilon$.
We performed all-electron self-consistent electronic structure calculations for each strain considered using the spin-polarized relativistic muffin-tin orbital method [9]. We obtained the magnetocrystalline anisotropy via the force-theorem, i.e., defined the magnetocrystalline anisotropy energy as the difference in the eigenvalue sum between the spin-polarized relativistic band structures for the different magnetization directions considered. Previous theoretical and numerical investigations (see Ref. [8] and references therein) show that the force theorem should be a good approximation to fully self-consistent total energy calculation of anisotropy energy in both second and fourth order in the spin-orbit coupling. The number of $\mathrm{k}$ points over the irreducible wedge (IW) of the Brillouin zone (BZ) used in the self-consistent calculations was around 7000 over $\frac{1}{8} \mathrm{BZ}$ for the $\left[\begin{array}{lll}1 & 1 & 0\end{array}\right]$ orientation, 3800 over $\frac{1}{16}$ for the $\left[\begin{array}{lll}0 & 0 & 1\end{array}\right]$ orientation and 2673 over $\frac{1}{12}$ for the $\left[\begin{array}{lll}1 & 1 & 1\end{array}\right]$ direction. For the adequate calculation of the magnetocrystalline anisotropy energy a denser $k$-mesh in the IW of the Brillouin zone is required. The number of $k$ points over the IW used was therefore approximately 66000 over $\frac{2}{8} \mathrm{BZ}$ for the $\left[\begin{array}{lll}1 & 1 & 0\end{array}\right]$ orientation, 53000 over $\frac{2}{16}$ of the BZ for the $\left[\begin{array}{lll}0 & 0 & 1\end{array}\right]$ orientation and 39000 over $\frac{3}{12}$ of the BZ for the [ $\left.\begin{array}{lll}1 & 1 & 1\end{array}\right]$ direction. Further computational details can be found in Refs. $[7,8]$.

Fig. 1 shows the calculated anisotropy constant $\left(K_{\text {out }}\right)$ (Fig. 1a) and the corresponding magnetoelastic coupling constant $\left(B_{2}\right)$ (Fig. 1c) of FCC Co as a function of the lateral strain for the $\left[\begin{array}{lll}1 & 1 & 1\end{array}\right]$ orientation. Also displayed in Fig. 1 are $B_{1}$ and $B_{2}$ that are derived from the calculated

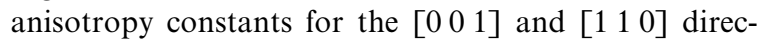
tions [8]. The perpendicular and in-plane anisotropy constants, $K_{\text {out }}$ and $K_{\text {in }}$, for the [ $\left[\begin{array}{lll}1 & 1 & 0\end{array}\right]$ direction of FCC $\mathrm{Ni}$ are displayed as function of the lateral strain in Fig. 2a

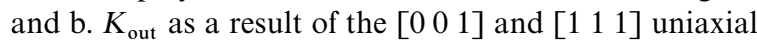
strains are also shown in Fig. 2. Finally, the associated magnetoelastic coupling constants, $B_{1}$ and $B_{2}$ of the strained FCC Ni, are displayed in Fig. 3. Since most FCC $\mathrm{Co}$ and $\mathrm{Ni}$ films are grown on a substrate which has a slightly larger in-plane lattice constant (e.g., $\mathrm{Cu}$ ), we limited ourselves to the consideration of extensive lateral strain regimes, i.e., compressive strains along the film normal. The compressive strain perpendicular to the layer was determined by the lateral strain such that the unit-cell volume remained constant. For simplicity, strain will always be referred to the elastic lateral strain in the rest of this paper unless stated otherwise. In the strained FCC Co (Ni) films, the unit-cell volume is generally different from that of bulk FCC Co (Ni). However, the volume changes for the $\mathrm{Co}$ and $\mathrm{Ni}$ films on, e.g., $\mathrm{Cu}$ are estimated to be within $1 \%$ and thus we neglect their effects in this paper.

Fig. 1 shows that for the $\mathrm{Co}\left[\begin{array}{lll}1 & 1 & 1\end{array}\right]$ orientation, $K_{\text {out }}$ prefers the perpendicular magnetization (positive). The $K_{\text {out }}$ initially increases linearly with the strain up to 


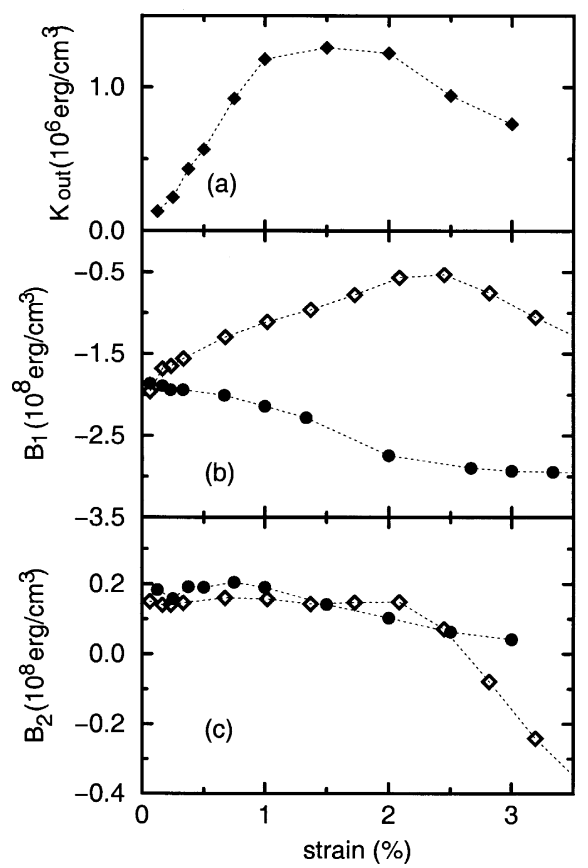

Fig. 1. Out-of-plane anisotropy constant $K_{\text {out }}$ for a strained FCC Co as a function of strain in the (1 1111$)$ plane (a). Magnetoelastic coupling constant $B_{1}(\mathrm{~b})$ and $B_{2}$ (c) for a strained FCC Co as a function of strain. Open diamonds in (b) and (c) are for strain in the $\left(\begin{array}{lll}1 & 1 & 0\end{array}\right)$ plane, solid circles in (b) for the $\left(\begin{array}{lll}1 & 1 & 0\end{array}\right)$ plane and in (c) for the $\left(\begin{array}{lll}1 & 1 & 1\end{array}\right)$ plane. The lines are a guide to the eye only.

$1 \%$, becomes nearly constant as the strain further increases and then decreases steadily beyond $2 \%$. This interesting result suggests that $B_{2}$ is almost constant only up to the strain of $1 \%$ (Fig. 1c). As the strain goes from $1 \%$ to $3 \%$, the $B_{2}$ drops dramatically from $0.20 \times 10^{8}$ to $0.04 \times 10^{8} \mathrm{erg} / \mathrm{cm}^{3}$. Also shown in Fig. $1 \mathrm{c}$ is the $B_{2}$ for the $\left[\begin{array}{lll}1 & 1 & 0\end{array}\right]$ direction which, on the other hand, remains more or less unchanged for the strain up to $2 \%$. Beyond the strain of $2 \%$, the $B_{2}$ decreases steadily and changes sign at the strain of $2.6 \%$. Strikingly, the $B_{1}$ for the Co $\left[\begin{array}{lll}1 & 1 & 0\end{array}\right]$ and Co $\left[\begin{array}{lll}0 & 0 & 1\end{array}\right]$ depend dramatically not only on the strain size but also on the uniaxial direction (see Fig. 1b). In particular, the magnitude of the $B_{1}$ for the Co $\left[\begin{array}{lll}1 & 1 & 0\end{array}\right]$

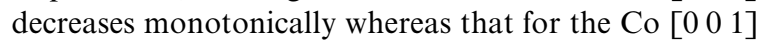
increases steadily up to the strain of about $2.5 \%$. For example, at $2.5 \%$ strain, the $B_{1}$ for the $\left[\begin{array}{lll}1 & 1 & 0\end{array}\right]$ and $\left[\begin{array}{lll}0 & 0 & 1\end{array}\right]$ are, respectively, $-0.6 \times 10^{8}$ and $-2.8 \times 10^{8} \mathrm{erg} / \mathrm{cm}^{3}$, compared with about $-2 \times 10^{8} \mathrm{erg} / \mathrm{cm}^{3}$ at the zero strain limit.

Note that the calculated $B_{1}$ for the Co [ $\left[\begin{array}{lll}1 & 1 & 0\end{array}\right]$ and $\left[\begin{array}{lll}0 & 0 & 1\end{array}\right]$ directions agree well at the small strains (see Fig. 1b), as they should. So are the calculated $B_{2}$ for the Co $\left[\begin{array}{lll}1 & 1 & 0\end{array}\right]$ and [ [ 1111$]$ directions. This is satisfactory. Furthermore, the calculated $B_{1}$ at the smallest strains

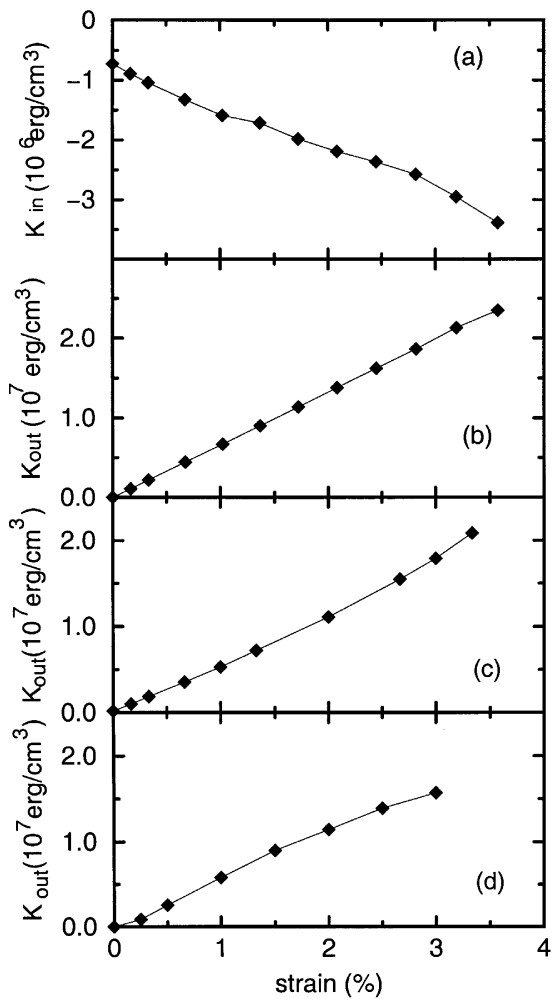

Fig. 2. In-plane anisotropy constant $K_{\text {in }}$ (a), out-of-plane anisotropy constant $K_{\text {out }}$ (b), for a strained FCC Ni as a function of strain in the $\left(\begin{array}{lll}1 & 1 & 0\end{array}\right)$ plane. Out-of-plane anisotropy constant $K_{\text {out }}$ for a strained FCC Ni as a function of strain in the $\left(\begin{array}{lll}0 & 0 & 1\end{array}\right)$ plane (c), and in the ( $\left.\begin{array}{lll}1 & 1 & 1\end{array}\right)$ plane (d). The lines are a guide to the eye only.

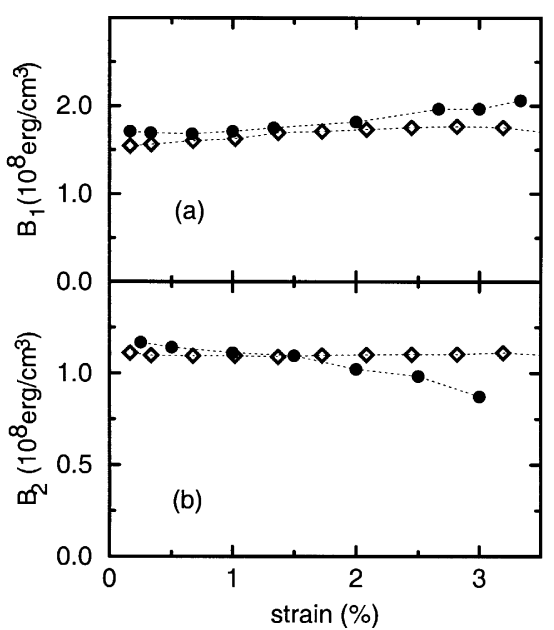

Fig. 3. Calculated magnetoelastic coupling constant $B_{1}$ (a) and $B_{2}$ (b) for a strained FCC $\mathrm{Ni}$ as a function of strain. Open diamonds are for strain in the (1 110$)$ plane, solid circles in (a) for the $\left(\begin{array}{lll}1 & 1 & 0\end{array}\right)$ plane and in (b) for the (lllll) plane. The lines are a guide to the eye only. 
used are $-1.9 \times 10^{8} \mathrm{erg} / \mathrm{cm}^{3}$ for the Co $\left[\begin{array}{lll}1 & 1 & 0\end{array}\right]$ and $-2.0 \times 10^{8} \mathrm{erg} / \mathrm{cm}^{3}$ for the Co $\left[\begin{array}{lll}0 & 0 & 1\end{array}\right]$, both being in good agreement with a previous experiment $\left(-1.6 \times 10^{8} \mathrm{erg} / \mathrm{cm}^{3}\right)[10]$ and a recent calculation $\left(-1.6 \times 10^{8} \mathrm{erg} / \mathrm{cm}^{3}\right)[5]$ by using a more accurate fullpotential method. However, no measurement on the value of FCC Co $B_{2}$ has been reported, though the value derived by extrapolating the measurements on FCC Co-Pd alloys $\left(2.6 \times 10^{8} \mathrm{erg} / \mathrm{cm}^{3}\right)$ [4] would suggest the above theoretical $B_{2}\left(0.2 \times 10^{8} \mathrm{erg} / \mathrm{cm}^{3}\right)$ being one order of magnitude too small.

In contrast to the case of FCC Co, the calculated anisotropy constants $\left(K_{\text {out }}, K_{\text {in }}\right)$ of strained FCC Ni for all the three [ $\left[\begin{array}{lll}1 & 1 & 0\end{array}\right],\left[\begin{array}{lll}0 & 0 & 1\end{array}\right]$ and $\left[\begin{array}{lll}1 & 1 & 1\end{array}\right]$ directions considered suggest a much smaller dependence of $B_{1}$ and $B_{2}$ on orientation (see Fig. 2). Indeed, Fig. 2 shows that the calculated $K_{\text {out }}$ and $K_{\text {in }}$ increases nearly linearly with strain for each direction. As a result, the $B_{1}$ and $B_{2}$ remain almost constant for strain up to $2 \%$ (see Fig. 3) and change discernably only beyond this strain region. The calculated $B_{1}$ and $B_{2}$ at the small strains agree with experiments at least in sign. The $B_{1}$ and $B_{2}$ derived from the measured magnetostrictions $\lambda_{001}$ and $\lambda_{00} 0_{1}$ and elastic constants $C_{11}, C_{12}$ and $C_{44}$ [11] are, respectively, 0.89 and $1.02 \times 10^{8} \mathrm{erg} / \mathrm{cm}^{3}$. The latter is in good agreement of the calculated $B_{2}$ of about $1.1 \times 10^{8} \mathrm{erg} / \mathrm{cm}^{3}$ while the former is about half of the calculated $B_{1}$ value of around $1.6 \times 10^{8} \mathrm{erg} / \mathrm{cm}^{3}$. This discrepancy in FCC Ni $B_{1}$ between experiments and theory is much reduced in a recent calculation using a more accurate full-potential method [12].

The author thanks Professor G.A. Gehring for stimulating discussions on the topics considered in this paper.
This work is supported by the National Science Council of ROC (NSC 88-2112-M002-0043). Part of the computational work was carried out on the computers at the National Center for High-Performance Computing, ROC.

\section{References}

[1] J.A.C. Bland, B. Heinrich (Eds.), Ultra-Thin Magnetic Structures I, Springer, Berlin, 1994.

[2] R. Jungblut, M.T. Johnson, J. aan de Stegge, A. Reinders, F.J.A. den Broeder, J. Appl. Phys. 75 (1994) 6424.

[3] R. Naik, A. Poli, D. McKague, A. Lukaszew, L.E. Wenger, Phys. Rev. B 51 (1995) 3549.

[4] G. Bochi, O. Song, R.C. O’Handley, Phys. Rev. B 50 (1994) 2043.

[5] R. Wu, A.J. Freeman, J. Appl. Phys. 79 (1996) 6209.

[6] J. Fassbender, G. Guntherodt, C.H. Mathieu, B. Hillebrands, R. Jungblut, J. Kohlhepp, M.T. Johnson, D.J. Roberts, G.A. Gehring, Phys. Rev. B 57 (1998) 5870.

[7] G.Y. Guo, J. Magn. Magn. Mater. 176 (1998) 97.

[8] G.Y. Guo, D.J. Roberts, G.A. Gehring, Phys. Rev. B 59 (1999) 14466.

[9] H. Ebert, Phys. Rev. B 38 (1988) 9390.

[10] R.C. O'Handley, unpublished, cited in J. Fassbender, G. Guntherodt, C.H. Mathieu, B. Hillebrands, R. Jungblut, J. Kohlhepp, M.T., Johnson, D.J. Roberts, G.A. Gehring, Phys. Rev. B 57 (1998) 5870.

[11] P. Bruno, Physical Origins and Theoretical Models of Magnetic Anisotropy, Ferienkurse des Forschungszentrums Juelich, Juelich, 1993.

[12] R.Q. Wu, L.J. Chen, A. Shick, A.J. Freeman, J. Magn. Magn. Mater. 177-181 (1998) 1216. 